\title{
Public Decision Making
}

\section{Connecting Artificial Intelligence and Crowds}

\author{
Helen K. Liu \\ Department of Political Science, \\ National Taiwan University, Taipei, \\ Taiwan \\ helenliu4@gmail.com
}

\author{
MuhChyun Tang \\ Department of Library and \\ Information Science, National Taiwan \\ University, Taipei, Taiwan \\ mctang@g.ntu.edu.tw
}

\author{
Kuang-hua Chen \\ Department of Library and \\ Information Science, National Taiwan \\ University, Taipei, Taiwan \\ khchen@ntu.edu.tw
}

\begin{abstract}
The recent breakthrough of artificial intelligence, as well as the wide adoption of the wisdom of the crowd, also known as collective intelligence, across sectors, has received attention and excitement across disciplines. In addition to the scientific breakthrough, recent public sector studies recognize AI's potential contributions in public services, such as big data for decision making, the development of smart cities, and social and health care. Studies have also recognized crowdsourcing's potential for service provisions, innovation, information generation, and policymaking. However, we have only a limited understanding of the connections between these two types of intelligence and adoption conditions to properly utilize them for the public sector. To understand what roles AI and crowds can play in enhancing public services and policymaking, we adopt a bibliometric analysis to identify emerging themes and interconnections between these two streams of literature. Our study provides key themes and significance for each cluster. Our first examination of $\mathrm{AI}$ and crowd literature regarding connection to public values, complementary in public decision making, as well as future potential for joint adoption by governments provides some implications for future considerations.
\end{abstract}

\section{CCS CONCEPTS}

- Computing/technology policy; - Cross-computing tools and techniques; $\bullet$ Semantics and reasoning;

\section{KEYWORDS}

Coword analysis, Literature-based discovery, Artificial intelligence, Crowdsourcing, Public sector

\section{ACM Reference Format:}

Helen K. Liu, MuhChyun Tang, and Kuang-hua Chen. 2020. Public Decision Making: Connecting Artificial Intelligence and Crowds. In The 21st Annual International Conference on Digital Government Research (dg.o '20), June 15-19, 2020, Seoul, Republic of Korea. ACM, New York, NY, USA, 9 pages. https://doi.org/10.1145/3396956.3396965

Permission to make digital or hard copies of part or all of this work for personal or classroom use is granted without fee provided that copies are not made or distributed for profit or commercial advantage and that copies bear this notice and the full citation on the first page. Copyrights for third-party components of this work must be honored For all other uses, contact the owner/author(s).

dg.o '20, June 15-19, 2020, Seoul, Republic of Korea

(c) 2020 Copyright held by the owner/author(s).

ACM ISBN 978-1-4503-8791-0/20/06.

https://doi.org/10.1145/3396956.3396965

\section{INTRODUCTION}

Crowdsourcing was developed to address complex decision-making processes and tasks [1]. Crowdsourcing is defined as an online open call for a collective of individuals to solve problems or perform tasks. Additionally, artificial intelligence, while receiving much new attention, is a much older concept from the 1950s that refers to machines mimicking human cognitive functions, such as learning and problem solving. In the public sector, crowdsourcing has been adopted for problem solving [2,3], information generation [4], and service provision [5, 6], while AI has been highly referenced for decision making [7], the Internet of Things (IoT) [8], big data [9], and smart cities [10]. However, the developments of both types of intelligence have evolved and coevolved in recent decades. For instance, hybrid collective intelligence has been developed to resolve auto-driving, distance and name record searches [11]. Additionally, both types of intelligence have gained recognition for their recent progress. With the progress of $\mathrm{AI}$ in assembling and mimicking human cognition functions, to what extent can AI and crowdsourcing (collective human intelligence) complement each other? What is the progress of their new development? What are the challenges ahead for their adoption for public decision making?

The purpose of this study is to identify ways in which both AI and crowdsourcing can inform public decision-making processes while providing a systematic review of the recent development of a hybrid AI crowd, with a focus on new progress and common areas of these two streams of literature. More specifically, we conduct a systematic literature review of journal articles addressing these two topics within the literature of public administration. A total of 187 articles published between 1986 and 2019 were included in our analysis. By applying co-word analysis in bibliometrics, we were able to identify how each public decision-making tool has been applied and how they can complement each other. Using a social network analytical-based community detection method, we were able to identify different emerging themes related to each technique (see, for example, [12]-[14]).

Revealing these themes allows both academics and practitioners 1) to gain new insights on public decision making based on the state-of-art as well as what have been learned of the two fields; 2) to reveal conditions, opportunities and challenges for adoptions of these two types of intelligent systems; and 3) to propose future research agendas.

\section{RELEVANT LITERATURES}

Recently, several essential review articles have discussed the potentials, threats, and implications of AI $[15,16]$ and crowdsourcing $[4,5,17]$. For instance, Agarwal [15] notes that "the pace of change 


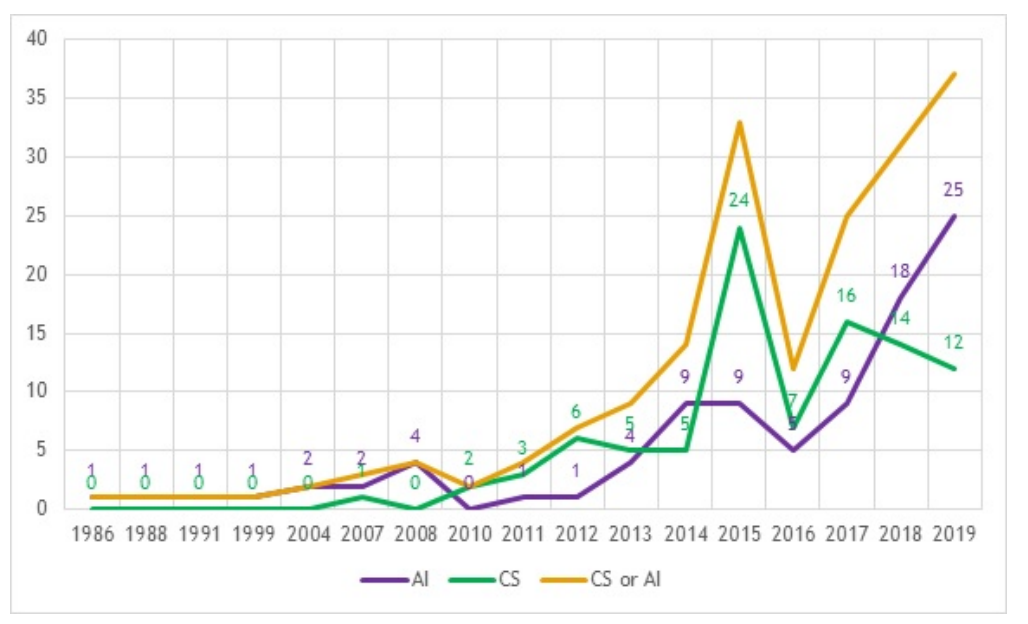

Figure 1: The growth in the AI and crowdsourcing literature from 1986 to 2019

outside our public policy institutions is faster than the pace of change within." He also discusses the impacts of AI on public services and administration in terms of key areas, including electronic government services, jobs, economies, social safety nets, revenue shortfalls, consumer protection, and privacy. He strongly advocates that core public agencies need to reveal "the unreviewed or unvalidated use of pretrained models, AI systems licensed from third-party vendors, and algorithmic processes created in-house" according to the AI Now Institute (p920, [15]).

More recently, Bullock [16] further discussed how AI could complement humans to reduce street-level bureaucrats' discretion in bureaucracy. Through a literature review, he showed that AI dominates in areas where the tasks require high analyzability, have a lower level of complexity and uncertainty, and few deviations when compared to humans. However, humans dominate in areas where the tasks have higher complexity and uncertainty, with many deviations, but relatively lower analyzability. He also argues that with the advancement of AI, learning AI could also handle tasks with a high level of uncertainty.

Liu [5] discussed the implications of adopting crowdsourcing from three dimensions: aligning, motivating and evaluating. In particular, Liu [5] suggested six lessons for crowdsourcing adoption: (1) aligning solutions with targeted public problems, (2) aligning tasks with participants' capacities, (3) incentivizing participants with rewards, (4) enhancing the quality of contribution through learning and skill-building, (5) empowering participants through peer review, and (6) integrating a reputation system in the process. Others viewed crowdsourcing as public policy instruments, namely, virtual labor markets, tournament crowdsourcing, and open collaboration $[4,17]$. They argued that the selection of various crowdsourcing modes should depend on cost, anonymity, scale of crowd, IT structure, time required to implement, task magnitude, and reliability of the crowd [4]. While those studies provide useful lessons for AI or crowdsourcing adoptions, there has been little discussion about these two types of intelligence (artificial vs. crowd). Therefore, this study intends to apply bibliometrics to explore emerging themes and potentials for utilizing both types of intelligence.

\section{DATA AND METHODS}

A keyword co-occurrence analysis of articles on the topic of crowdsourcing and AI published in the domain public administration was adopted and was supplemented by expert interpretation to reveal the major research themes related to either policy tool and how they might complement each other. Social network analytical techniques were then applied to the co-word network for each topic, followed by a network that identified that connected these two topics.

\subsection{Data}

To compare the use of $\mathrm{AI}$ and crowdsourcing as a decision-making tool in the public administration literature, a literature searches that combined journal and keyword searches of Scopus, one of the leading citation databases, was performed. Morphological and semantic variations of the two keywords were searched (See Appendix I for the queries used), limited to the journal titles belonging to the public administration domain, which consists of a set of 130 journals sorted under the public administration category by either Scopus or Google Scholar (see Appendix II for the list of journals included). Author-assigned keywords in articles in the search results were then extracted, along with their co-occurrence data so co-word network analysis could be applied.

\subsection{Network Construction}

Co-word analysis has been long used in bibliometrics to study the intellectual structure of a domain (See for example [12, 13]). Only author-assigned keywords were used to construct the coword networks. Vocabulary control was performed by a domain expert to normalize spelling variations and synonyms of the same concept (see Appendix II for the thesaurus). From a total of 655 distinct keywords appearing in the search results, 601 concepts were identified initially after vocabulary control, which constituted the node of the co-word network. Whenever a pair of keywords appeared in the same articles, a linkage was established between them based on which a network of keywords could be constructed. 


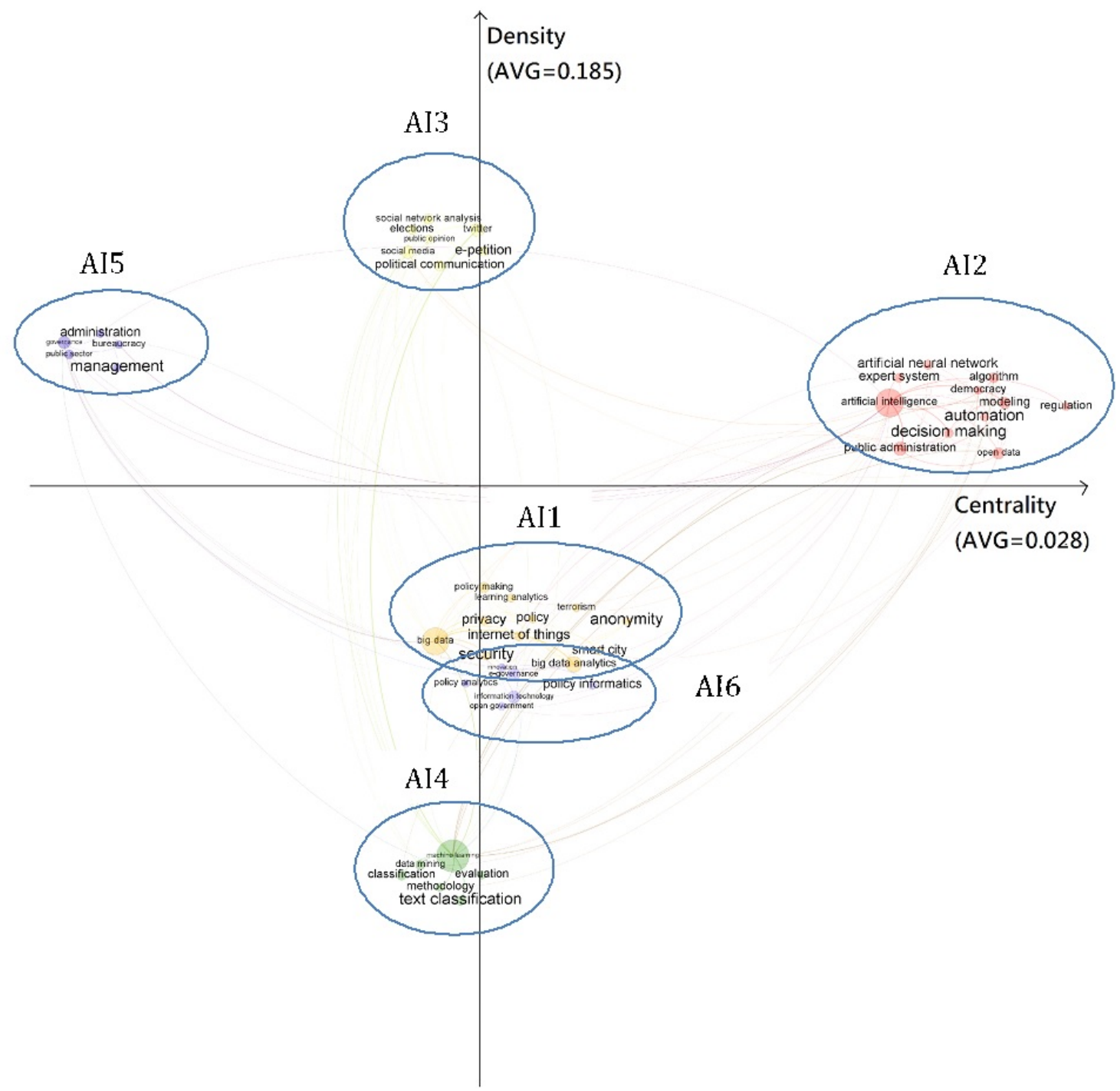

Figure 2: Concept map of literature in the field of AI

The bibliometric mapping software VOS viewer was used for visualizing the results [18], where each concept constituted a node in the network, while the edge weight was determined by their association strength, which normalizes the strength of pairwise keyword co-occurrence by the frequency of each keyword [19]. To further rule out the chance occurrence of concepts, a threshold of concept co-occurrence larger than 1 was applied in all the co-occurrence networks reported in the results section. The modularity-based community detection method in network analysis [20] was applied to identify major research themes in either topic.

\section{RESULTS}

\subsection{Overall Trends}

Figure 1 illustrates the growth in the AI and CI literature from 1986 to 2019 in public administration-related journals. According to the trend lines, there was a sharp growth in the AI literature from 2016 and a jump between 2014 and 2016 for CI studies.

\subsection{AI}

We first analyzed the topics separately, first AI, then crowdsourcing. The modularity analysis of the AI co-word network produced six main clusters of concepts. Each cluster of the concepts can be seen as a cohesive subject area, which were then projected on a strategic diagram [21].

In a strategic diagram, the $\mathrm{x}$-axis represents the centrality of a subject area; the higher the centrality, the stronger the connections it has with other subject areas. The higher the centrality of a subject, the more well-connected it is to other subjects. The y-axis represents the within-group density, which measures the cohesiveness or interconnectedness within a subject area. The higher the density 


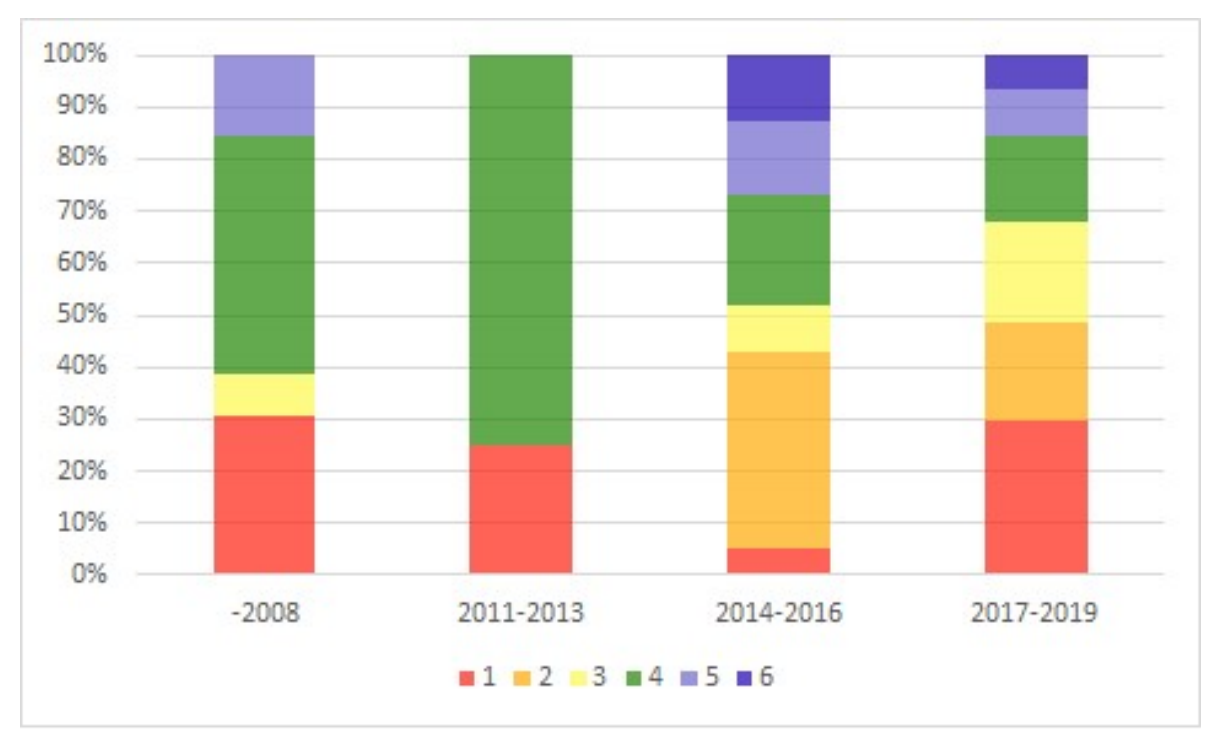

Figure 3: Trends in AI clusters

within a subject, the denser the linkages of the keywords within a subject. The origins of the coordinate are the mean centrality and density of the subjects in respective topics.

As shown in Figure 2, the two largest and most representative, marked as AI1 and AI2, refer to policymaking and automation in decision making. In AI1, big data and analytics have high frequencies within the policymaking areas. In AI2, AI and algorithms have high frequencies within the public administration for automation. In particular, AI2 not only has strong connections within the group but also with the other groups. In other words, AI2 shares strong connections with other groups. AI3, which refers to elections and public opinion, has impacts on public preferences and applications from social media and Twitter usages. Connecting with other groups, AI4 with implications from machine learning and text classification refers to methodology in the field. Less connected with the other groups, AI5 refers to conceptual discussions on AI management or AI impacts on public administration. AI6 refers to an emerging area on policy informatics.

Figure 3 shows the publications in different subject areas over time. Since 2008, the overall development of the AI literature has become more diverse. Since 2014, two new emerging areas have been developed: AI automation and policy informatics. In particular, the AI automation literature addresses the intervention of $\mathrm{AI}$ on public administration decision making as well as the roles of humans and machines. For instance, Wagner [11] discussed the role of human control over automated decision-making processes in self-driving cars, searches on passenger name records and social media content. He further argued that there is a lack of regulation on decisions made by both humans and machines. To maximize the mixed human and automated systems, Wagner [11] made the following criteria for accountable quasi-automation: (1) amount of time, (2) degree of qualification, (3) degree of liability, (4) level of support, (5) adaptation, (6) access to information, and (7) agency.

\subsection{Crowdsourcing}

Figure 4 shows seven main clusters. The two largest and most representative, marked as CS1 and CS2, refer to governance and public opinion. In CS1, emphasis is placed on adopting crowdsourcing for governance with a specific focus on collective action, legislation, red tape of the bureaucracy, volunteering in the public sector, and privacy and security. In CS2, key concepts reflected the importance of democratic innovation and deliberative democracy in the process of policymaking and understanding public opinion through open data, open source and improvement of survey research through the adoption of Mechanical Turk. While CS1 and CS2 refer to more generalized public administration concepts, CS3 through CS7 refer to more specific areas. For instance, while CS3 refers to open innovation and coproduction and has impacts on smart cities through social participation, CS4 focuses on responsiveness from governments. CS5 through CS7 emphasize theoretical contributions in strategy for citizen engagement, transparency in the election process, and open collaboration through the online labor market.

Figure 5 shows the trends of CS cluster development. Again, the body of the CS literature has grown into different focuses. In particular, two emerging areas developed after 2014, including CS2 and CS7. These emerging areas demonstrate the potential of CS for participatory democracy and democratic innovation. CS2, in particular, emphasizes engaging crowds in the city planning of City of Palo Alto [22], law reforms in Finland [23, 24], and institutional designs on democratic innovation through cases documented on Participedia [25]. Connected with CS2, studies from CS7 emphasize the development of a typology for policy crowdsourcing, including virtual labor markets, tournament crowdsourcing, and open collaboration $[4,17]$.

\subsection{AI U Crowdsourcing}

Figure 6 represents the concepts that most frequently co-occurred (at least two times) in the overall set of journal articles in the fields of 


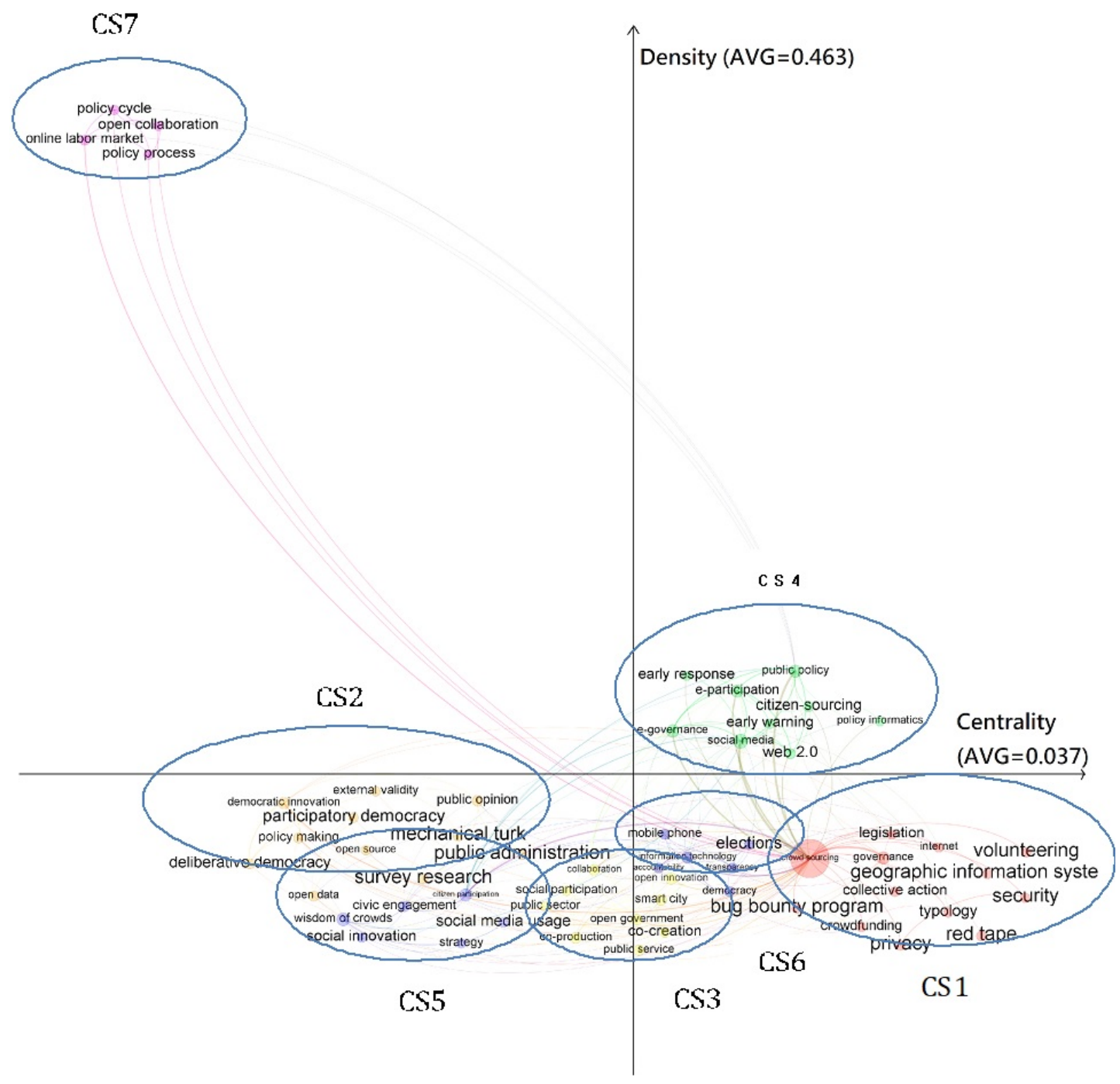

Figure 4: Concept map of literature in the field of crowdsourcing

AI and crowdsourcing; this is done to help identify areas of research each decision technique can complement each other [26]. In the figure, the clusters of concepts provide potential links between the two areas of research, including a cluster of 102 co-occurring concepts. In particular, the figure shows a cluster of concepts connecting social media, big data and innovation. Common interconnections between the two bodies of literature and emerging themes include governance, open government, open data, election, policymaking, e-governance, policy informatics, and smart city. In particular, the most recent developments are on big data, smart cities, and China, measured by the average year of the published dates.

Figure 7 shows nine main clusters that indicate the potential of joint development between AI and CS. Three of the largest and most representative, marked as $\mathrm{AC} 1, \mathrm{AC} 2$, and $\mathrm{AC} 3$, refer to governance, automation in decision-making, and political communication. A cluster closely connected to $\mathrm{AC} 3, \mathrm{AC} 4$, refers to online campaigns and policymaking through big data. Another closely connected cluster, AC5 and AC6, refers to policy analytics and government responsiveness. $\mathrm{AC} 7$, with a strong connection to $\mathrm{AC} 2$, refers to smart city and social innovation. Less connected to any group, AC8 and AC9 represent policy design and methodological approach. Additionally, AC6 to AC8 contain concepts mostly developed from the CS literature only.

Figure 8 shows the trends of AI U CS cluster development. Again, the body of the literature has grown into different streams. In particular, two emerging areas, AC4 and AC7, developed after 2011, while AC8 and AC9 developed after 2014. These emerging areas demonstrate the potential of adopting AI and CS. AC4 shows the potential for adopting big data and analytics for understanding elections, policymaking, and online campaigns as well as engaging the public through deliberative democracy. AC7 shows the emerging importance of smart cities and the adoption of social innovation 


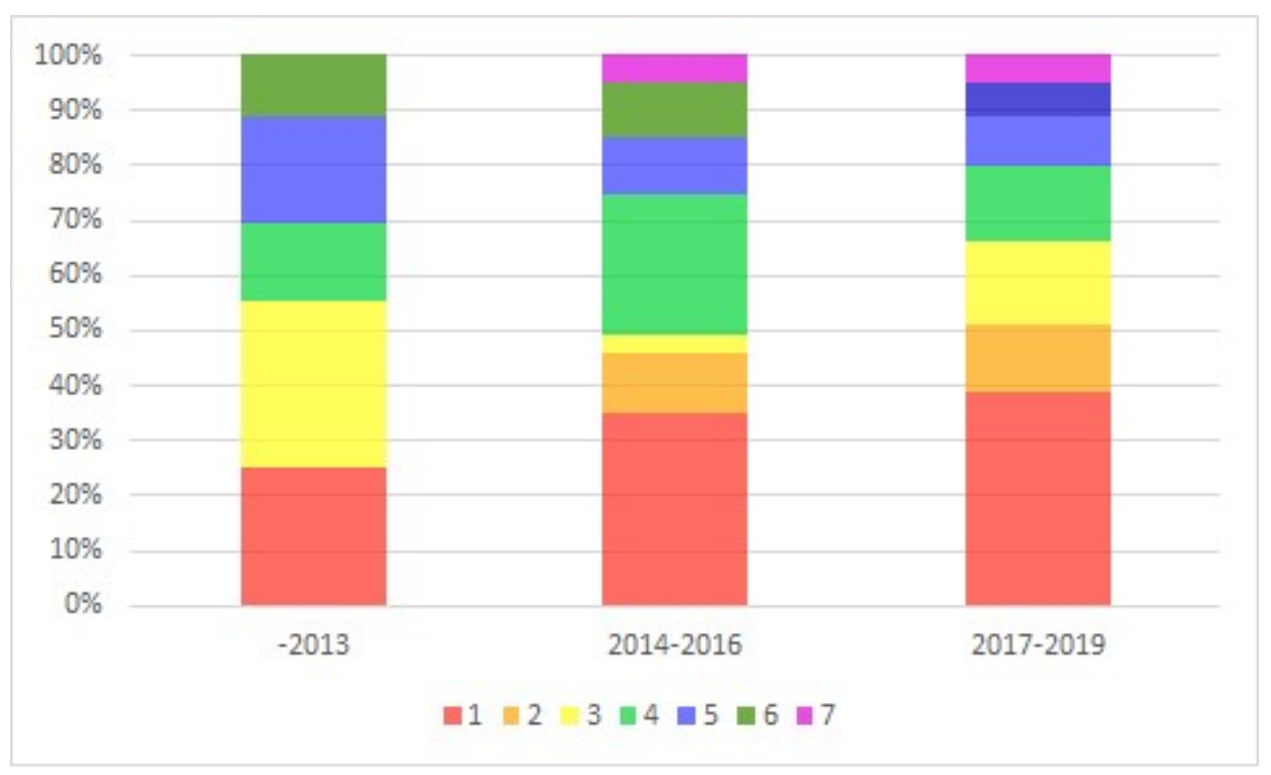

Figure 5: Trends in crowdsourcing clusters

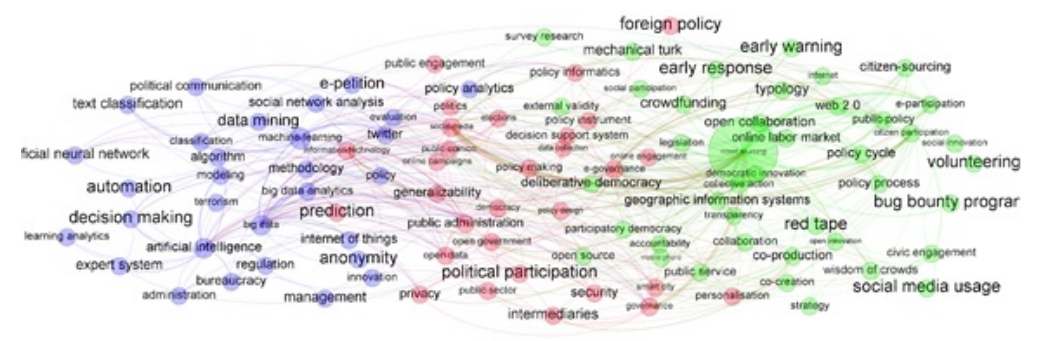

Figure 6: Concept map of literature in the field of both AI and crowdsourcing

through technologies. AC8 and AC9 both show the importance of exploring new policy instruments and research methodologies in the public administration field, given the development of new technologies.

\section{DISCUSSIONS}

Different but related research areas emerge from these in-depth analyses of the co-word concepts from the literature in AI and crowdsourcing. Figure 7 shows the two separate clusters of literature from AI and CS. However, in Figure 8 of the clustering analysis, $\mathrm{AC} 1$ and $\mathrm{AC} 4$ are among the concepts attracting interdisciplinary scholars.

\subsection{Creating Public Values}

Introducing new technology into the public sector requires changes in values and thus create new technology-driven paradigms [27]. Additionally, public values are important outcomes from how technologies are socially accepted [28]-[30]. Our data show implications from the development of $\mathrm{AI}$ and CS evolve around the core values of public administration, as reflected through each cluster in Figure 7. Our co-word analysis illustrates important public values that have been discussed extensively, including accountability, transparency, democracy, deliberative and participatory democracy, privacy, citizen participation, responsiveness, openness, and collaboration. In particular, citizen participation and democracy can be enhanced by AI through diffused political participation [31] as well as by crowdsourcing as engagement tools [23, 32]. Emphasizing public value creations makes AI and crowdsourcing literature in the public sector distinct from the private sector.

\subsection{Towards New Hybrid Intelligence}

Our analysis shows how both intelligent systems can complement each other to enhance public decision making. For instance, our reviewed studies show new development of AI in healthcare [33], egovernment [34], and citizen-government communication through chatbots [35], and the Internet of Things [36], as well as widely adoption of crowdsourcing in the governments [3]-[5, 37]. For instance, citizens' complaints through 311 services have been utilized 


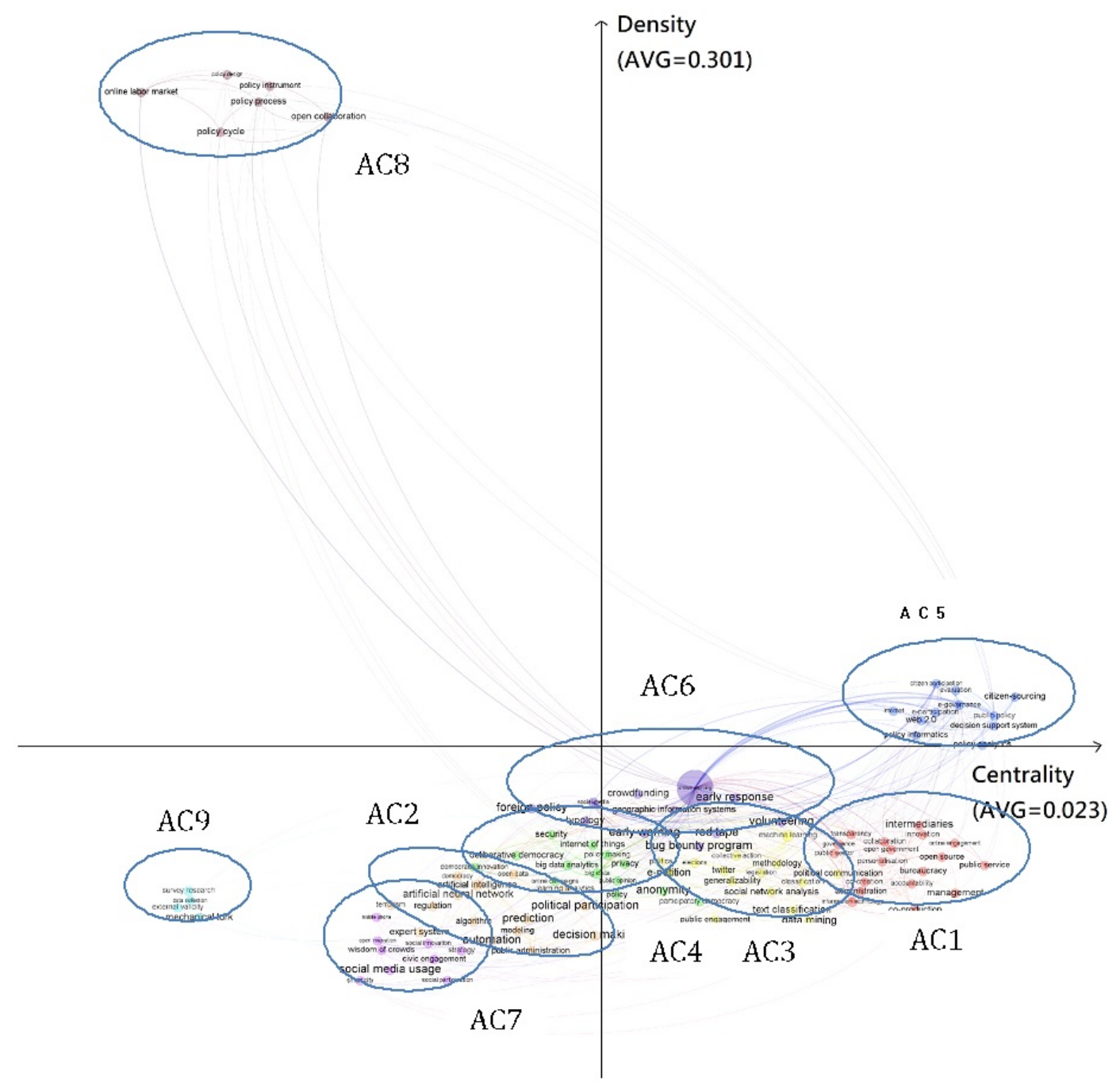

Figure 7: Concept map of literature in the field of both AI and crowdsourcing with color coded clusters

for improving city management decisions [38]. With the insertions of chatbots or AI systems into citizen hotline services [39], analyses have accounted for these new interventions from AI before utilizing those crowdsourcing data.

Furthermore, emerging hybrid intelligence have been built on a solid body of literature, including human-computer interaction [40], hybrid human-machine [41, 42], collective hybrid intelligence [43], and hybrid machine-crowd interaction [40]. This stream has made new progress in recent years, and multiple frameworks have been developed to maximize the benefits of both intelligences while addressing their limitations. For instance, improvements have been made for the optimization of hybrid systems [41], which address the traditional limitations of AI or CS and improve the accuracy of decision-making. Public decisions on traffic control, disease prevention, and human resources in the public sector, to name just a few, would benefit from redesigning existing frameworks and regulations to accommodate those new developments.

\subsection{Future Potentials of AI and Crowdsourcing}

Our review shows that AI has created great potential to improve the efficiency and effectiveness of governmental services; however, as pointed out by Mehr [39], AI decisions require human inputs to mitigate ethical risks. Emerging hybrid intelligence should be further understood and explored, particularly in the policy informatics area. Additionally, a hybrid citizen hotline service, health care, and transportation system received the most attention from our studied literature. Potential areas that will require further theoretical development include online deliberative engagement platforms, online regulation systems, and other hybrid systems for improving democratic systems.

Specifically, from the strategic map (Figure 7), one of the potential interconnections between $\mathrm{AI}$ and crowdsourcing addresses conceptual development for AI and CS in governance. In particular, studies in this cluster show how emerging technologies could 


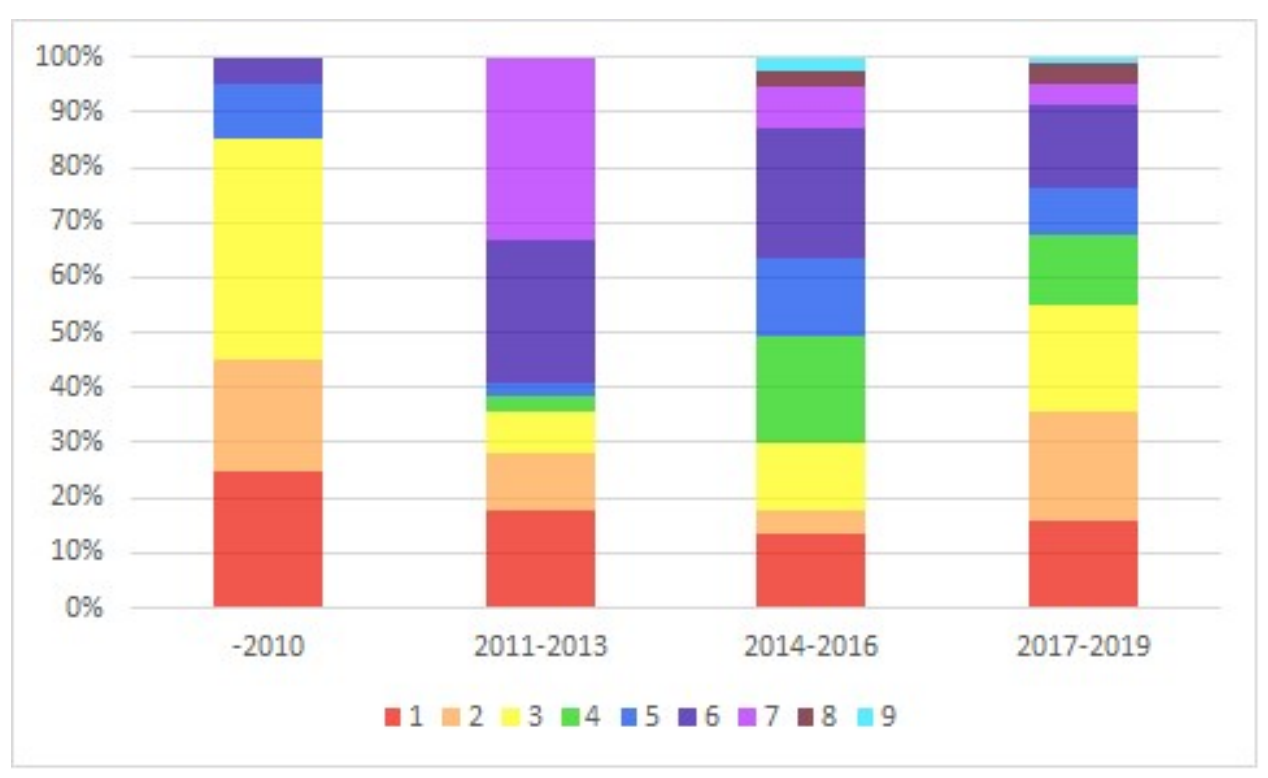

Figure 8: Trends in AI and crowdsourcing joint clusters

consolidate open government [44], enhance effective public services [5], and create public values [30]. Another interconnection addresses online campaigns and policymaking processes through the utilization of big data $[45,46]$ and the emphasis of participatory democracy [22].

Second, based on centrality (between-group connections) and density (within-group connection) in Figure 7, AC2 and AC7 occupied a space jointly. While AC2 is predominately formed by concepts from the AI literature, AC7 is dominated by concepts from the CS literature. Given that those two clusters have connections, there is potential for future development. For instance, Piscopo et al. [47] innovatively built a model to predict the sense of community and participation through a machine learning method to open government data. Through the model, one can predict community and local capacity by utilizing various social and economic developmental open government data. Adopting machine learning to utilize open data has implications for predicting the acceptance and adaptation of digital cities as well [10].

\section{CONCLUSIONS}

By systematically analyzing the converging areas between AI and CS from the literature published in public administration-related journals. The preliminary results show that the literature on AI and CS in public administration is inseparable from essential public value creation. Additionally, a solid body of literature addresses how $\mathrm{AI}$ and CS are complementary, and additional regulations should address those hybrid systems. Furthermore, our co-word analyses show potential for the future development of AI and CS, including areas of automation in government and smart city development as well as political communication and online campaigns. Meanwhile, potential methodological and policy instrumental designs are emerging areas that deserve attention from public administration and political science scholars.
This kind of exploration between literature in the AI and CS fields is still much in the early stage, and much more has yet to be done. Much more also needs to be known about the technological implications on the potential areas. However, this study has provided a descriptive basis for additional research. There is a continuing need for an adequate theoretical basis or framework for the practical selection of AI and CS for public decision making. In particular, future studies should focus on governance, policymaking, and smart cities where both AI and CS scholars share common interests.

\section{ACKNOWLEDGMENTS}

Our gratitude goes to Chen Ya-Chi and Lai Ching-Yi for their research assistance. This research project was supported by Yushan (Young) Scholar Award, 2018-2023, Ministry of Education, Taiwan, R.O.C. [Project No.: 2018-08-01-2023-07-31] and the Ministry of Science and Technology, Taiwan, R.O.C. [Project No. MOST1082410-H-002-011- and MOST 108-2638-H-002 -001 -MY2].

\section{REFERENCES}

[1] Howe, J. 2006. The rise of crowdsourcing. Wired magazine, 14, 6, 1-4.

[2] Brabham, D. C. 2009. Crowdsourcing the public participation process for planning projects. Planning Theory, 8, 3 (July 2009), 242-262.

[3] Ines Mergel and Kevin C. Desouza. 2013. Implementing open innovation in the public sector: The case of challenge.gov. Public Adm. Rev. 73, 6 (Oct. 2013), 882-890. DOI: https://doi.org/10.1111/puar.12141

[4] John Prpić, Araz Taeihagh, and James Melton. 2015. The fundamentals of policy crowdsourcing. Policy Internet 7, 3 (Aug. 2015), 340-361. DOI: https://doi.org/10. 1002/poi3.102

[5] Helen K. Liu. 2018. Crowdsourcing government: Lessons from multiple disciplines. Public Adm. Rev.77, 5 (Jul. 2018), 656-667. DOI: https://doi.org/10.1111/puar.12808

[6] M. Jae Moon. 2018. Evolution of co-production in the information age: Crowdsourcing as a model of web-based co-production in Korea. Policy Society 37, 3 (Sep. 2018), 294-309. DOI: https://doi.org/10.1080/14494035.2017.1376475

[7] Tambe, P., Cappelli, P. and Yakubovich, V. 2019. Artificial intelligence in human resources management: challenges and a path forward. California Management Review, 61, 4 (August 2019), 15-42. 
[8] Jenifer Winter. 2015. Algorithmic discrimination: Big data analytics and the future of the internet. In: Jenifer Winter and Ryota Ono, editors. Public Administration and Information Technology. Springer International Publishing, Cham, 125-140. DOI: https://doi.org/10.1007/978-3-319-22994-2 8.

[9] Fred Phillips. 2017. A perspective on 'Big Data'. Sci. Public Policy 44, 5 (Apr. 2017), 730-737. DOI: https://doi.org/10.1093/scipol/scx012

[10] Leonidas G. Anthopoulos and Theologis E. Tougountzoglou. A viability mode for digital cities: Economic and acceptability factors. In: Christopher G. Reddick and Stephen K. Aikins, editors. Web 2.0 Technologies and Democratic Governance. Public Administration and Information Technology. Springer, New York, NY, 79-96. DOI: https://doi.org/10.1007/978-1-4614-1448-3_6

[11] Ben Wagner. 2019. Liable, but not in control? Ensuring meaningful human agency in automated decision-making systems. Policy Internet 11, 1 (Jan. 2019), 104-122. DOI: https://doi.org/10.1002/poi3.198

[12] Muh-Chyun Tang, Weijen Teng, and Miaohua Lin. 2019. Determining the critical thresholds for co-word network based on the theory of percolation transition. $f$. Doc.76, 2 (Dec. 2019), 462-483. DOI: https://doi.org/10.1108/jd-06-2019-0117

[13] Muh-Chyun Tang, Yun Jen Cheng, and Kuang Hua Chen. 2017. A longitudinal study of intellectual cohesion in digital humanities using bibliometric analyses. Scientometrics. 113, 2 (May 2017), 985-1008. DOI: https://doi.org/10.1007/s11192017-2496-6

[14] Romo-Fernández, L.M., Guerrero-Bote, V.P. and Moya-Anegón, F. 2013. Co-word based thematic analysis of renewable energy (1990-2010). Scientometrics, 97, 3 (April 2013), 743-765.

[15] Agarwal, P.K. 2018. Public administration challenges in the world of AI and Bots. Public Administration Review, 78, 6 (Aug 2018), 917-921.

[16] ullock, J.B. 2019. Artificial intelligence, discretion, and bureaucracy. The American Review of Public Administration, 49, 7 (June 2018), 751-761.

[17] Araz Taeihagh. 2017. Crowdsourcing: A new tool for policy-making? Policy Sci.50, 4 (Nov. 2017), 629-647. DOI: https://doi.org/10.1007/s11077-017-9303-3

[18] Nees Jan van Eck and Ludo Waltman. 2009. Software survey: VOSviewer, a computer program for bibliometric mapping. Scientometrics. 84, 2 (Dec. 2009), 523-538. DOI: https://doi.org/10.1007/s11192-009-0146-3

[19] Nees Jan van Eck and Ludo Waltman. 2009. How to normalize cooccurrence data? An analysis of some well-known similarity measures. 7. Am. Soc. Inf. Sci. Technol.60, 8 (Aug. 2009), 1635-1651. DOI: https://doi.org/10.1002/asi.21075

[20] Vincent D. Blondel, Jean-Loup Guillaume, Renaud Lambiotte, and Etienne Lefebvre. 2008. Fast unfolding of communities in large networks. F. Stat. Mech. Theory Exp.2008, 10 (Oct. 2008), P10008. DOI: https://doi.org/10.1088/1742-5468/2008/10/ p10008

[21] Michel. Callon, Jean. Pierre. Courtial, and Françoise Laville. 1991. Co-word analysis as a tool for describing the network of interactions between basic and technological research: The case of polymer chemsitry. Scientometrics. 22, 1 (Sep. 1991), 155-205. DOI: https://doi.org/10.1007/bf02019280

[22] Kaiping Chen and Tanja Aitamurto. 2019. Barriers for Crowd's impact in crowdsourced policymaking: Civic data overload and filter hierarchy. Int. Public Manag 7.22, 1 (Oct. 2019), 99-126. DOI: https://doi.org/10.1080/10967494.2018.1488780

[23] Tanja Aitamurto and Hélène Landemore. 2016. Crowdsourced deliberation: The case of the law on off-road traffic in Finland. Policy Internet 8, 2 (Apr. 2016), 174-196. DOI: https://doi.org/10.1002/poi3.115

[24] Henrik Serup Christensen, Maija Karjalainen, and Laura Nurminen. 2015. Does crowdsourcing legislation increase political legitimacy? The case of avoin ministeriö in Finland. Policy Internet 7, 1 (Mar. 2015), 25-45. DOI: https://doi.org/10. $1002 /$ poi3.80

[25] Graham Smith, Robert C. Richards, and John Gastil. 2015. The potential of participedia as a crowdsourcing tool for comparative analysis of democratic innovations. Policy Internet 7, 2 (May 2015), 243-262. DOI: https://doi.org/10.1002/poi3.93

[26] Don R. Swanson. 1986. Fish oil, Raynaud's syndrome, and undiscovered public knowledge. Perspect. Biol. Med.30, 1 (Autumn 1986), 7-18. DOI: https://doi.org/10. 1353/pbm.1986.0087

[27] Frank Bannister and Regina Connolly. 2014. ICT, public values and transformative government: A framework and programme for research. Gov. Inf. Q.31, 1 (Jan 2014), 119-128. DOI: https://doi.org/10.1016/j.giq.2013.06.002
[28] Mark H. Moore. 1995. Creating Public Value: Strategic Management in government. Harvard University Press, Cambridge, MA.

[29] Antonio Cordella and Carla M. Bonina. 2012. A public value perspective for ICT enabled public sector reforms: A theoretical reflection. Gov. Inf. Q.29, 4 (Oct. 2012), 512-520. DOI: https://doi.org/10.1016/j.giq.2012.03.004

[30] J. Ignacio Criado and J. Ramon Gil-Garcia. 2019. Creating public value through smart technologies and strategies. Int. F. Public Sect. Manag.32, 5 (Jul. 2019), 438-450. DOI: https://doi.org/10.1108/ijpsm-07-2019-0178

[31] Savaget, P., Chiarini, T. and Evans, S. 2019. Empowering political participation through artificial intelligence. Science and Public Policy, 46, 3 (June 2019), 369-380.

[32] Mukhametkaliyeva, Y. and Bastaubayeva, A., 2018. Crowdsourcing as a Public Engagement Tool in Rulemaking Activities of Kazakhstan. Public administration issues, 3, 138-163.

[33] Tara Qian Sun and Rony Medaglia. 2019. Mapping the challenges of Artificial Intelligence in the public sector: Evidence from public healthcare. Gov. Inf. Q.36, 2 (Apr. 2019), 368-383. DOI: https://doi.org/10.1016/j.giq.2018.09.008

[34] Chih-Hao Ku and Gondy Leroy. 2014. A decision support system: Automated crime report analysis and classification for e-government. Gov. Inf. Q.31, 4 (Oct. 2014), 534-544. DOI: https://doi.org/10.1016/j.giq.2014.08.003

[35] Aggeliki Androutsopoulou, Nikos Karacapilidis, Euripidis Loukis, and Yannis Charalabidis. 2019. Transforming the communication between citizens and government through AI-guided chatbots. Gov. Inf. Q.36, 2 (Apr. 2019), 358-367. DOI: https://doi.org/10.1016/j.giq.2018.10.001

[36] Samir Chatterjee, Jongbok Byun, Kaushik Dutta, Rasmus Ulslev Pedersen, Akshay Pottathil, and Harry Xie. 2018. Designing an Internet-of-Things (IoT) and sensor-based in-home monitoring system for assisting diabetes patients: Iterative learning from two case studies. Eur. 7. Inf. Syst.27, 6 (Jul. 2018), 670-685. DOI: https://doi.org/10.1080/0960085x.2018.1485619

[37] Taewoo Nam. 2012. Suggesting frameworks of citizen-sourcing via Government 2.0. Gov. Inf. Q.29, 1 (Jan. 2012), 12-20. DOI: https://doi.org/10.1016/j.giq.2011.07. 005

[38] Akemi Takeoka Chatfield and Christopher G. Reddick. 2018. Customer agility and responsiveness through big data analytics for public value creation: A case study of Houston 311 on-demand services. Gov. Inf. Q.35, 2 (Apr. 2018), 336-347. DOI: https://doi.org/10.1016/j.giq.2017.11.002

[39] Hila Mehr. 2017. Artificial Intelligence for Citizen Services and Government. Harvard Kennedy School, Ash Center for Democratic Governance and Innovation, Cambridge, MA.

[40] Filipa Correia, Francisco S. Melo, and Ana Paiva. 2019. Group intelligence on social robots. In2019 14th ACM/IEEE International Conference on Human-Robot Interaction (HRI). Daegu, Korea (South), 703-705. DOI: https://doi.org/10.1109/ HRI.2019.8673089.

[41] Dominik Dellermann, Philipp Ebel, Matthias Söllner, and Jan Marco Leimeister. 2019. Hybrid intelligence. Bus. Inf. Syst. Eng.61, 5 (Mar. 2019), 637-643. DOI: https://doi.org/10.1007/s12599-019-00595-2

[42] Besmira Nushi, Ece Kamar, and Eric Horvitz. 2018. Towards accountable ai: Hybrid human-machine analyses for characterizing system failure. In Sixth AAAI Conference on Human Computation and Crowdsourcing; 2018.

[43] Morteza Moradi, Mohammad Moradi, Farhad Bayat, and Adel Nadjaran Toosi. 2019. Collective hybrid intelligence: Towards a conceptual framework. Int. F. Crowd Sci.3, 2 (Aug. 2019), 198-220. DOI: https://doi.org/10.1108/ijcs-03-2019-0012

[44] Jeremy Millard. 2017. European strategies for e-governance to 2020 and beyond. In: Adegboyega Ojo and Jeremy Millard, editors. Government 3.0 - Next Generation Government Technology Infrastructure and Services. Public Administration and Information Technology. Springer International Publishing, Cham, 1-25. DOI: https://doi.org/10.1007/978-3-319-63743-3 1.

[45] Irina Pencheva, Marc Esteve, and Slava Jankin Mikhaylov. 2018. Big data and AIA transformational shift for government: So, what next for research? Public Policy Adm.35, 1 (Jun. 2018), 24-44. DOI: https://doi.org/10.1177/0952076718780537

[46] Paola Mavriki and Maria Karyda. 2019. Big data in political communication: Implications for group privacy. Int. F. Electron. Gov.11, 3/4 (Nov. 2019), 289-309. DOI: https://doi.org/10.1504/ijeg.2019.103716

[47] Alessandro Piscopo, Ronald Siebes, and Lynda Hardman. 2017. Predicting sense of community and participation by applying machine learning to open government data. Policy Internet 9, 1 (Mar. 2017), 55-75. DOI: https://doi.org/10.1002/poi3.145 